\title{
Utility of the Easy-Care Standard 2010 in the Comprehensive Geriatric Assessment of Adults Aging with Developmental Disabilities
}

\author{
Ellen Kaehr, MD \\ Department of Geriatrics, Indiana University School of Medicine, Indianapolis, IN \\ Patricia Abele, FNP-BC, Milta Little, DO \\ Division of Geriatric Medicine, Saint Louis University School of Medicine, St. Louis, MO
}

To the Editor:

“Older people seldom have only medical or social need.”

Ian Philp

Anyone working with aging adults knows the physical, mental, and social needs of this population are great. Independence and well-being depend on a strong foundation in all three of these health domains. ${ }^{1}$ The lack of one standardized instrument to efficiently assess and address the three domains led to the development of the Easy-Care model in 1994 by Philp and colleagues. The components of the Easy-Care model were gathered from a variety of validated tools using a consensus process by health professionals in Europe. In the original acceptability studies, the model was found to be useful for the office setting, particularly to identify levels of unmet need for community-dwelling elders. ${ }^{1 ;}{ }^{2}$ Systematic interventions to address these needs

This is the author's manuscript of the article published in final edited form as:

Kaehr, E., Abele, P., \& Little, M. (2016). Utility of the Easy-Care Standard 2010 in the Comprehensive Geriatric Assessment of Adults Aging with Developmental Disabilities. Journal of the American Medical Directors Association, 17(12), 1159-1160. https://doi.org/10.1016/j.jamda.2016.08.024 
can help to prevent or delay institutionalization and reduce hospitalization. ${ }^{3}$ International studies consistently show that community-dwelling elders have difficulty with moving outside one's home, housework, shopping, and looking after one's own health. Cognitive impairment, loneliness, and depression were also identified frequently using the Easy-Care Standard $2010 .^{4}$ Participants had a greater use of home care and day care resources. ${ }^{5}$ The original model was modified to the more streamlined Easy-Care Standard 2010 after early acceptability and reliability trials.

This version is a three-part questionnaire with 49 core questions. The aim is to obtain a broader picture of outpatient geriatric needs by assessing seven areas: communication, taking care of oneself, transportation, safety, living arrangements, ability to stay healthy, and mental health. ${ }^{4}$ Its use is limited in the post-acute and residential care facilities, as the few studies that exist for this patient population did not show a significant benefit over the Resident Assessment Instrument. Overall, Easy-Care Standard 2010 was found to have good international acceptability, feasibility, usability, cost-effectiveness, and validity. There is limited evidence for its reliability, which may limit its use for population-based screening of geriatric syndromes and frailty until further evidence becomes available. $^{5}$

At Saint Louis University, we applied the Easy-Care 2010 model to screen community-dwelling older adults aging with developmental disabilities to identify threats to independence. People with developmental disabilities are similar to the general population in that they are living longer and are therefore at risk of developing the same age-related diseases and treatment complications. However, individuals in this population face unique challenges as they age, such as increased vulnerability, difficult behaviors, pre-existing cognitive limitations, restricted living 
environments, and social stigmas. Our aim was to see if the Easy-Care Standard 2010 could be used as a part of the comprehensive geriatric assessment to capture additional needs in this unique aging population above those we traditionally see in our clinics. We partnered with the Saint Louis Association on Aging with Developmental Disabilities, which provides individualized support to community-dwelling adults with developmental disabilities, including cerebral palsy, mental retardation, epilepsy, head injuries, and autism. Although the Easy-Care model was designed to serve as a comprehensive geriatrics assessment, we added additional assessment tools to further quantify ability and need. We screened for cognitive impairment with the Saint Louis University Mental Status Examination (SLUMS), depression with the Patient Health Questionnaire (PHQ-9), fall risk with the Toulouse falls assessment, frailty with the FRAIL Scale, sarcopenia with the SARC-F, and risk for weight loss with the Simplified Nutritional Assessment Questionnaire (SNAQ). ${ }^{3 ; 4 ; 5 ; 6 ; 7 ; 8}$ Sixty-four $(\mathrm{N}=64)$ total assessments were completed over 3 years. Thirty-two were initial assessments, and 16 patients received follow-up assessments 2 to 3 years after the initial meeting. This research was approved by the Saint Louis University IRB and was performed under exemption status.

Of the 32 patients who received only an initial assessment, the average age was 58.4. The most common comorbidities included obesity (56.5\%), diabetes mellitus (37.5\%), arthritis (34\%), depression (28\%), and history of falls (25\%). As a result, common recommendations centered on maintaining a healthy weight via exercise (66\%), nutrition education (37.5\%), and weight loss (22\%). Also, 22\% of patients were advised to undergo polysomnography due to concern for obstructive sleep apnea. 
Falls were fairly prevalent (25\%) in this population aging with disabilities. Patients had moderately high Toulouse Falls Assessment scores, with an average of 18.3 out of 30. This scale was designed to identify correctable problems that lead to falls. Higher scores are better, and any missed points are potential areas for intervention.

Encouraging physical findings included a low prevalence of frailty (four patients) and sarcopenia (three patients). Essentially no patients were underweight, and the average SNAQ was high at 16.5, with 14 or below indicating a risk for weight loss. From a mental health standpoint, depression was a prevalent comorbidity (25\%), but the average PHQ-9 score was low at 3.5, providing evidence that depression is being addressed in this population. Other frequently overlooked areas of physical health in this population included dental care (37.5\%), eye care (12.5\%), skin care (12.5\%), and podiatry care (9\%).

Although the patients screened well in the physical domain, there was a high prevalence of Instrumental Activities of Daily Living (IADL) disability and therefore social need. Adding the Easy-Care Standard 2010 to the comprehensive geriatric assessments allowed us to identify these areas of need. Sixty-nine percent needed help with shopping, 62.5\% needed help navigating public transportation, $47 \%$ required assistance with housework, and $44 \%$ needed help in meal preparation and medication administration. These findings are consistent with prior studies of Easy-Care. $^{1 ; 2 ; 3}$

This high social support need was coupled with an alarmingly low average SLUMS score of 13.9 out of 30, with the cutoff for dementia being 19 or less in those with less than a high school education. Regardless of intellectual disability from developmental delay, much emphasis was placed on increasing mental activity via socialization (84\%) and cognitive stimulation therapy 
(CST) (72\%). The potential reversible causes of cognitive impairment such as polypharmacy, sensory deprivation, and depression were targeted with the recommendations of medication review (41\%), ENT referral (28\%), and psychotherapy referral (22\%).

In regard to the 16 patients who received two assessments, the average SLUMS was 16, falling into the category concerning for dementia. Of major concern, the SLUMS decreased by 2.2 points over a 2- to 3-year period. Another troubling observation was higher IADL dependence. Combining new and persistent deficits, 94\% of the patients needed help with public transportation, 81\% needed assistance shopping, and 60\% needed help with meal preparation. Again, the recommendations focused on preserving and improving brain function.

Similar to the first cohort, the follow-up cohort was obese and encouraged to exercise. As a result, 56\% of patients lost weight. About one-third of patients had an increase in their PHQ-9 scores, but the average score was 5 , still low and indicative of adequate treatment for depression. There was no major change in the Toulouse Falls Assessment, FRAIL Scale, and SARC-F.

Surprisingly, the physical disability in both cohorts was low. Of most concern was mental disability. We strongly advocate for cognitive screening when applying the Easy-Care model to those aging with developmental disabilities. With the progressive worsening of the SLUMS, one should evaluate for reversible causes of cognitive impairment, such as depression, hypothyroidism, folate or vitamin B12 deficiency, obstructive sleep apnea, polypharmacy, sensory deficit, chronic infection and CNS pathology. 9; 10;11;12

Overall, we found that the Easy-Care Standard 2010 coupled with cognitive testing is a helpful tool to identify threats to health, independence, and overall well-being in older adults aging with 
disabilities. Optimizing brain health with increased physical, mental, and social stimulation paired with local resources has the potential to create a meaningful impact in this population. 


\section{References}

1. Philp I. Can a medical and social assessment be combined? J R Soc Med 1997; 90(Suppl 32):11.

2. Philp I. Development of EASY-Care, for brief standardized assessment of the health and care needs of older people; with latest information about crossnational acceptability. J Am Med Dir Assoc 2014;15:42e46.

3. Melis RJ, Adang E, Teerenstra S, et al. Cost-effectiveness of a multidisciplinary intervention model for community-dwelling frail older people. J Gerontol Med Sci 2008;63A:275e282.

4. Talarska D, Pacholska R, Struga1a M, Wieczorowska-Tobis K. Functional assessment of the elderly with the use of EASY-Care Standard 2010 and Comprehensive Geriatric Assessment. Scand J Caring Sci 2016;30:419e426.

5. Craig C, Chadborn N, Sands G, et al. Systematic review of EASY-care needs assessment for community-dwelling older people. Age and Ageing 2015;44: 559e565.

6. Tariq SH, Tumosa N, Chibnall JT, et al. The Saint Louis University mental status (SLUMS) examination for detecting mild cognitive impairment and dementia is more sensitive than the mini-mental status examination (MMSE)ea pilot study. Am J Geriatr Psychiatry 2006;14:900e910.

7. Kroenke K, Spitzer RL, Williams JB. The Phq-9. J Gen Inter Med 2001;16: 606e613.

8. Morley JE, Rolland Y, Tolson D, Vellas B. Increasing awareness of the factors producing falls: The mini falls assessment. J Am Med Dir Assoc 2012;13:87e90.

9. van Kan GA, Rolland YM, Morley JE, Vellas B. Frailty: Toward a clinical definition. J Am Med Dir Assoc; 2008:71e72.

10. Woo J, Leung J, Morley JE. Validating the SARC-F: A suitable community screening tool for sarcopenia? J Am Med Dir Assoc 2014;15:630e634. 
11. Wilson MM, Thomas DR, Rubenstein LZ, et al. Appetite assessment: Simple appetite questionnaire predicts weight loss in community-dwelling adults and nursing home residents. Am J Clin Nutr 2005;82:1074e1081.

12. Cruz-Oliver DM, Malmstrom TK, Roegner M, et al. Cognitive deficit reversal as shown by changes in the Veterans Affairs Saint Louis University Mental Status (SLUMS) examination scores 7.5 years later. J Am Med Dir Assoc 2014;15:687. e5e687.e10. 